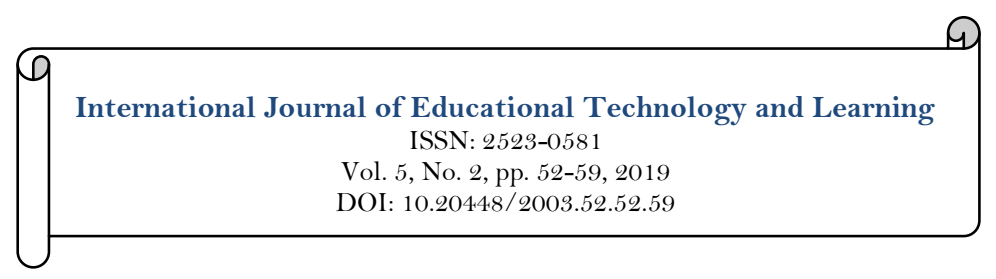

\title{
Serious Gaming as an Additional Learning Tool for Medical Education
}

\author{
Mehmet E. Aksoy ${ }^{1}$ \\ Mehmet E. Sayali ${ }^{2}$ \\ ${ }_{1.2}^{1 .}$ CASE Acibadem University Istanbul, Turkey \\ sEmail:merhans@gmail.com
}

\section{Abstract}

Computer-based learning methodologies have become more prevalent in the last decade. Web-based serious gaming and virtual patients are novel in medical education, which has the potential to become important tools to improve today's medical students' knowledge and performance. A total of 81 medical students participated in our study. They were either assigned to an intervention group or a control group. The intervention group completed a serious game module designed for Basic Life Support education. The control group received a theoretical lecture on the same content. On the next day, both groups received simulation-based hands-on training for BLS using the same criteria. All students were assessed with an Objective Structured Clinical Examination (OSCE) the following week. There was no difference between the intervention group using a web-based serious game module for $B L S$ and the control group that received a standard theoretical lecture from the faculty. Computer-based interactive serious games seem to present a favourable additional tool for medical education. OSCE results imply, that the use of serious games as a self-learning strategy can be as useful as theoretical lectures; which means that it is saving the time of learners and educators.
Keywords:

Medical education

Serious gaming

Web based learning

Basic life support

Licensed:

This work is licensed under a

Creative Commons Attribution 4.0

License.

Publisher:

Scientific Publishing Institute

Funding: This study received no specific financial support.

Competing Interests: The authors declare that they have no competing interests.

\section{Introduction}

According to Vygotsy, learning is acquired through cognitive, affective and psychomotor knowledge and skills (Vygotsky, 1978). Support through various resources and professionals eases learning. One such example occurs through classroom-based learning, which creates a one-to-one interaction between the teacher and the learner. In this approach, the learner gains the opportunity to repeat the training under the observation of the teacher and receives instant feedback to accomplish the learning objectives (Wilson \& Wittmann-Price, 2015). In this traditional approach, the instructor conducts the course according to a study program or a curriculum. The instructor also determines the structure of the lecture and the time allocation required for that specific material. For this reason, teachers are required to have effective writing and speaking skills, and they must be able to understand student's psychology during the teaching process. How new knowledge is acquired and existing knowledge is enhanced, depending on the use of various learning techniques. Each approach has its own advantages and disadvantages. One of the main disadvantages of traditional learning is the time constraint on the learning objectives (Dennen \& Burner, 2008). This problem might be counteracted by increasing the number of teaching hours or educators (Saini \& Wahid, 2014). This, however, increases the costs for educational institutions and learners.

For these reasons and with progress in technology new methodologies have been sought for. The Internet and the use of new information technologies lead to the advancement in learning and teaching methodologies that were adapted to the changing needs and expectations of an increasingly diverse population of learners 
belonging to a generational cohort called 'Millennials' or the 'Net Generation' (Baptista, 2011). Yolton and de Calesta foresaw a revolution in education that would lead to a shift from the traditional lecture style of information presented to a more active style of learning (Yolton \& Calesta, 2000). A reason for this paradigm shift is that in traditional learning, everyone has to learn the same thing at the same time, classes and training sessions are frustratingly hard for some and boringly easy for others' (Walz \& Deterding, 2014). Prensky concluded that game technologies can produce a great deal of learning and positive effects, 'such as quickly mastering and applying new skills and information, thinking laterally and strategically'(Prensky, 2012). Holtziger demonstrated the superiority of dynamic media over static textbook lessons (Holzinger, 2008). Experimental evidence have largely supported the effectiveness of the game-based learning approach over the years (Papastergiou, 2009).

Web-based education focuses on faster learning, reduced costs, increased access to learning materials and accountability for all participants in the learning process while it gives the learners the opportunity to schedule their working hours, social life and other needs (Halachev, 2009). Sending web-based self-learning material prior to simulation-based education allows participants to learn and also reduces educators' time used for theoretical education. Web-based learning enables access to knowledge, reduces the costs (Maloney et al., 2015) and faculty time, and also instructors become facilitators rather than teachers (Ruiz, Mintzer, \& Leipzig, 2006).

Although International cardiopulmonary resuscitation (CPR) guidelines' encourages the use of short video/computer self-learning courses with minimal or no instructor coaching (Perkins et al., 2015) to improve basic life support performance, and reports have shown advantages for using instructional video learning, comparison of different teaching methods is still rare in the literature.

All medical students in our university are being introduced to the general topic of Basic Life Support during their first year. Up to now, courses have been taught using a 2-hour theoretical lecture followed by 30 minutes per student hands-on training on a CPR-manikin. Considering the increasing use of computer-based education tools in medical education we decided to work on the development of a $3 \mathrm{D}$ interactive serious game for Basic Life Support at our University. This paper investigates the effectiveness of this web-based selflearning module in BLS compared to traditional lecture through objective structured clinical examination (OSCE).

\section{Methods}

\subsection{Virtual Learning Model}

All of the learning objectives were based on the ERC 2015 guidelines for Basic Life Support. The serious game was designed and developed in collaboration with the Department for Biomedical Device Technology of our University. The game opens with the starting page, where the participant may choose between the 'training ' and 'test 'option. The participant has to log in with his or her username and password. He or she can choose between English and Turkish versions of the game.

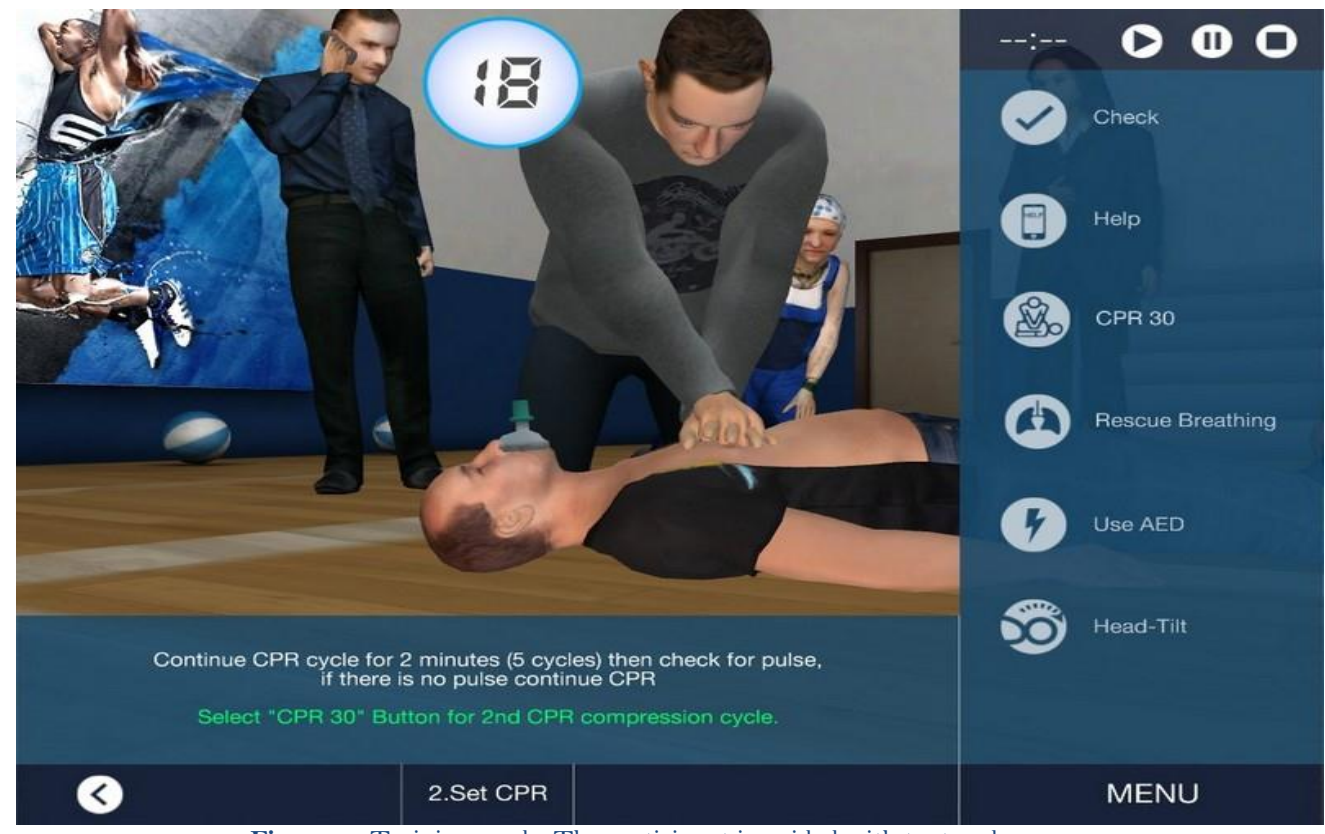

Figure-1. Training mode: The participant is guided with text and arrows.

Source: CASE- Acibadem University - own developed Serious Game. Figure presents a screen shot of this serious game. 
First, the participant is instructed to use the 'training 'mode, in which written instructions are given regarding the learning objectives and the structure of the game. In this training mode, the game is teaching the learner how to pass all demanded steps for successful resuscitation in an interactive $3 \mathrm{D}$ virtual environment with green highlighted text and blinking arrows they have to follow Figure 1. The Automated External Defibrillator (AED) is coming at the end of the $5^{\text {th }}$ cycle of the CPR and again the participant is guided with green highlighted text Figure 2. After completing the training session, the participants take the test mode, where they have to pass the same steps on their own without being instructed by the game. The results are given at the end of the game and are also automatically recorded with the username of the participant. Green stands for 'done well and in time', orange for 'done well but with delay' and red for 'missed actions'. The total time for using a serious game at home is not limited. Automated test results derived from the serious game of the participants in the investigation group were also analysed and compared with their OSCE scores.

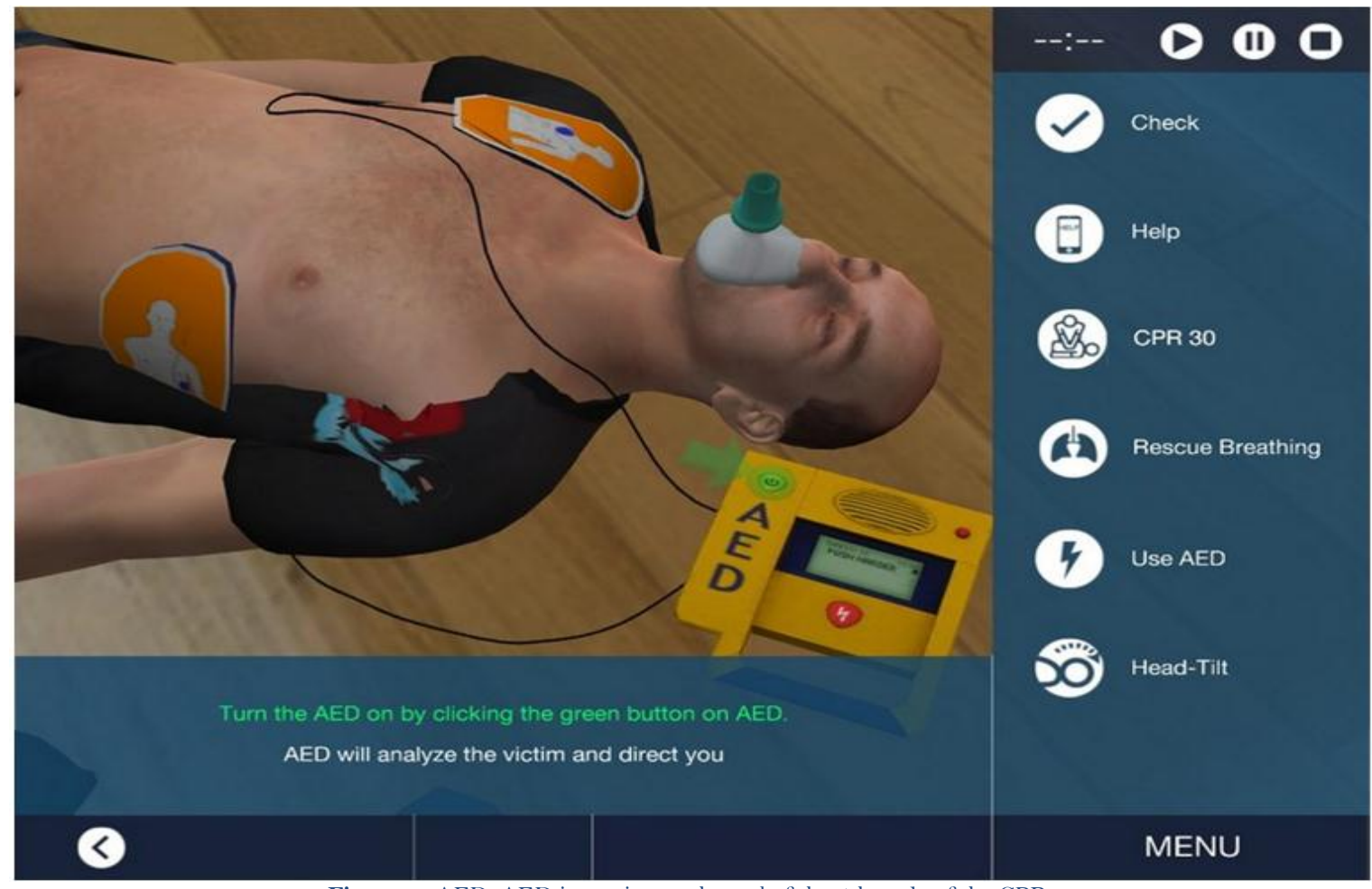

Figure-2. AED: AED is coming at the end of the 5th cycle of the CPR.

Source: CASE- Acibadem University- own developed Serious Game. Figure presents a screenshot of this serious game.

\subsection{Technical Features}

An interactive learning module compatible with multi-platform applications has been created. A virtual 3D simulation environment had to be developed. Unity was chosen as the $3 \mathrm{D}$ Visualization Engine due to its advantages in its licensing terms. Choosing the appropriate Learning Management System (LMS) and the integration of the Learning Management System were the other crucial decisions that were made while preparing this e-learning module. Moodle was selected as the Learning Management System (LMS) due to its advantage of being an open source system. ADL (Advanced Distributed Learning) was preferred as the Learning Record Store for the e-learning module. All data collected from the users were stored on a virtual server for further analysis. Design of our BLS- serious game sequence showing all procedural steps that have to be taken by the participant and the time limits to take these steps are shown in Table 1 . What makes this interactive serious gaming module different from existing solutions is that the trainees are able to watch any scene from any angle during gameplay by the help of a $3 \mathrm{D}$ visualization engine and are able to access their previous performances thanks to a dedicated learning management system with a learning record store running on a virtual server. All actions taken by the trainees and their timings are tracked using the SCORM (Sharable Content Object Reference Model) Tin Can standard, which also provides better data security and the ability to share data with other databases.

\subsection{Participants}

All participants were first-year medical students. They were about the same age and in equal distribution concerning gender, where else age and gender was not a variable in our investigation. They were divided into two groups. The control group received a standard traditional lecture-based course on BLS. In the intervention group, students were told to complete a web-based game training at home. They could choose 
when to start the session, they could repeat the training mode as often as it seems to be necessary for them and were able to stay linked to the computer game for as long as they needed. It was expected that they complete the test mode at least once before ending their session. On the next day, both groups received hands-on training for BLS on CPR manikins. One week later both groups were assessed using an OSCE.

\subsection{Study Design}

The study was approved by the Ethics committee of our University. A total of 90 first-year- medical students, all of them novices in BLS signed up in our study with their written consent. They were randomly assigned to an investigation group or a control group with 45 participants in each group. Since this was a study with voluntary participation, we had some dropouts due to personal reasons. Finally, 81 first yearmedical students participated in our study; 44 of them were in the intervention group and 37 in the control group. The intervention group completed a web-based serious game, that we had designed and developed for BLS education according to the ERC 2015 guidelines for Basic Life Support, while the control group received a theoretical lesson on the same content. On the next day, both groups received simulation-based hands-on training for BLS according to the same criteria. All students were assessed with an OSCE examination in the following week.

\subsection{Hands-on Training}

Hands-on training were done simulator based according to the ERC 2015 guidelines for BLS using CPR manikin. The course was given to all participants by the same educator. Participants were trained in a single rescuer scenario from finding an unresponsive person to the successful use of an Automated External Defibrillator. The educator provided immediate feedback on the participants' performance to ensure effective learning during the simulation. Concrete temporal recommendations for the procedural steps for CPR were:

1. Deliver compressions 'in the center of the chest'.

2. Compress to a depth of at least $5 \mathrm{~cm}$ but not more than $6 \mathrm{~cm}$.

3. Compress the chest at a rate of 100-120 / min. with as few interruptions as possible.

4. Allow the chest to recoil completely after each compression; do not lean on the chest.

The optimal total time was estimated to be 3 minutes for the whole sequence, from safety check to the use of the AED.

\subsection{Performance Observation Using OSCE}

Following the completion of both learning methods, each participant attended to an OSCE one week later. In this examination, participants experienced a cardiac arrest scenario. The participants were then requested to approach to the patient and to intervene as in a real-life situation. Performance observation was performed on all participants in a simulation-based session by two impartial examiners who had an inter-rater correlation of 0.82. Participants' performance was observed for ten subsequent criteria:

1. Ensure that the scene is safe

2. Check responsiveness by shaking gently and shouting loudly

3. Open the airway using the head tilt and chin lift technique

4. Calling for help

5. Sending someone for fetching AED

6. Starting and continuing high-quality CPR in 30 compressions and 2 ventilations sequence

7. Attaching the AED correctly when it arrives

8. Following the instructions are given by the AED

9. Delivering shock when demanded by the AED and to ensure all rescuers are not in contact with the patient prior to defibrillation

10. Continue with CPR for another 2 minutes or until the patient starts breathing

Performance observation as a quantitative approach was done using the aforementioned skill testing sheet consisting of ten items of critical CPR steps based on the 2015 ERC BLS Guidelines. To evaluate students' psychomotor skills we used the BLS scoring system of our simulation centre. Three medical specialists prepared this checklist via Delphi methodology, and two simulation instructors individually marked the performance of each participant based on their observations. Table 1 expresses the details of this checklist, which is identical to the aforementioned scoring system of our serious game.

Successful completion of each step was graded as 10 points, whereas any partial attempt and any insufficient step received 5 and o points respectively. The OSCE results were graded out of 100 points, and the time-based steps were evaluated based on the following criteria:

- Rescue breath durations should be equal to 1 (inhalation) and 1.5 (exhalation) seconds.

- Consciousness and breathing should be determined within 15 seconds.

- Chest compression should occur at a rate of 100-120/min.

- Re-evaluation should occur every 2 minutes (5 cycles) 
Table-1. Numerical score system for CPR performance as well as for our BLS- Serious Game scoring system.

\begin{tabular}{|c|c|c|}
\hline Skill component & & ived \\
\hline \multirow[b]{3}{*}{ Safety } & Correct & 10 \\
\hline & Insufficient or late & 5 \\
\hline & Not performed & 0. \\
\hline \multirow[b]{3}{*}{ Checked Consciousness ( verbal / pain ) } & Correct & 10 \\
\hline & Insufficient or late & 5 \\
\hline & Not performed & O. \\
\hline \multirow[b]{3}{*}{ Head Tilt / Check for breathing } & Correct & 10 \\
\hline & Insufficient or late & 5 \\
\hline & Not performed & $\mathrm{O}$. \\
\hline \multirow[b]{3}{*}{ Called For Help/call for AED } & Correct & 10 \\
\hline & Insufficient or late & 5 \\
\hline & Not performed & O. \\
\hline \multirow[b]{3}{*}{ Effective chest compression } & Correct & 10 \\
\hline & Insufficient or late & 5 \\
\hline & Not performed & O. \\
\hline \multirow{3}{*}{$\begin{array}{l}\text { Chest compression / Breathing rate } \\
30 / 2\end{array}$} & Correct & 10 \\
\hline & Insufficient or late & 5 \\
\hline & Not performed & O. \\
\hline \multirow[b]{3}{*}{ AED place Pads } & Correct & 10 \\
\hline & Insufficient or late & 5 \\
\hline & Not performed & 0. \\
\hline \multirow[b]{3}{*}{ AED On } & Correct & 10 \\
\hline & Insufficient or late & 5 \\
\hline & Not performed & $\mathrm{O}$. \\
\hline \multirow[b]{3}{*}{ AED Shock / Stand clear } & Correct & 10 \\
\hline & Insufficient or late & 5 \\
\hline & Not performed & O. \\
\hline \multirow{3}{*}{$\begin{array}{l}\text { Continue CPR for another } 2 \text { minutes or } \\
\text { until the patient starts breathing }\end{array}$} & Correct & 10 \\
\hline & Insufficient or late & 5 \\
\hline & Not performed & 0. \\
\hline
\end{tabular}

\section{Results}

81 first-year medical students were randomized into an intervention group and a control group. 44 students received web-based education with a serious game, while 37 students participated in a traditional lecture on BLS. The data analyst was blinded to which method of learning the students had received. 27 students in the intervention group $(60 \%)$ and 23 students in the control group $(62 \%)$ were female. Mean age of the students participating in web-based education was 19 (SD: 0.62) compared to 19.6 (SD: 0.69) in the control group.

Statistics were done using T-Test for two independent samples. Power analysis revealed that our sample size is sufficient for a 2 sample non-inferiority trial with 08.power at an equivalence margin of $10 \%$ in accordance with our pointing system. There was no significant difference between the intervention group completing a self-educational training and control group, which received a standard theoretical lecture on OSCE results. That means that the learning performance of the group who received a standard theoretical lecture of 2 hours and the performance of the group completing self-educational training on serious games was comparable. Mean OSCE examination score for the intervention group of 44 students was 88.65 , while the control group had a mean OSCE score of 88.78 , with a T-value is -0.04861 and the p-value is .96135.

As a secondary result, our serious gaming modules automated test results from the participants in the investigation group were very similar to their OSCE scores assessed by our instructors. After processing these data by using Pearson Correlation the $\mathrm{r}$ value was calculated as 0,8412 showing a strong correlation between the participants serious game and OSCE scores. $\mathrm{P}$ value was calculated as $\mathrm{p}=0,00001$ at the significance level 0,01 . P value also revealed the strong significance between these data Figure 3 . 


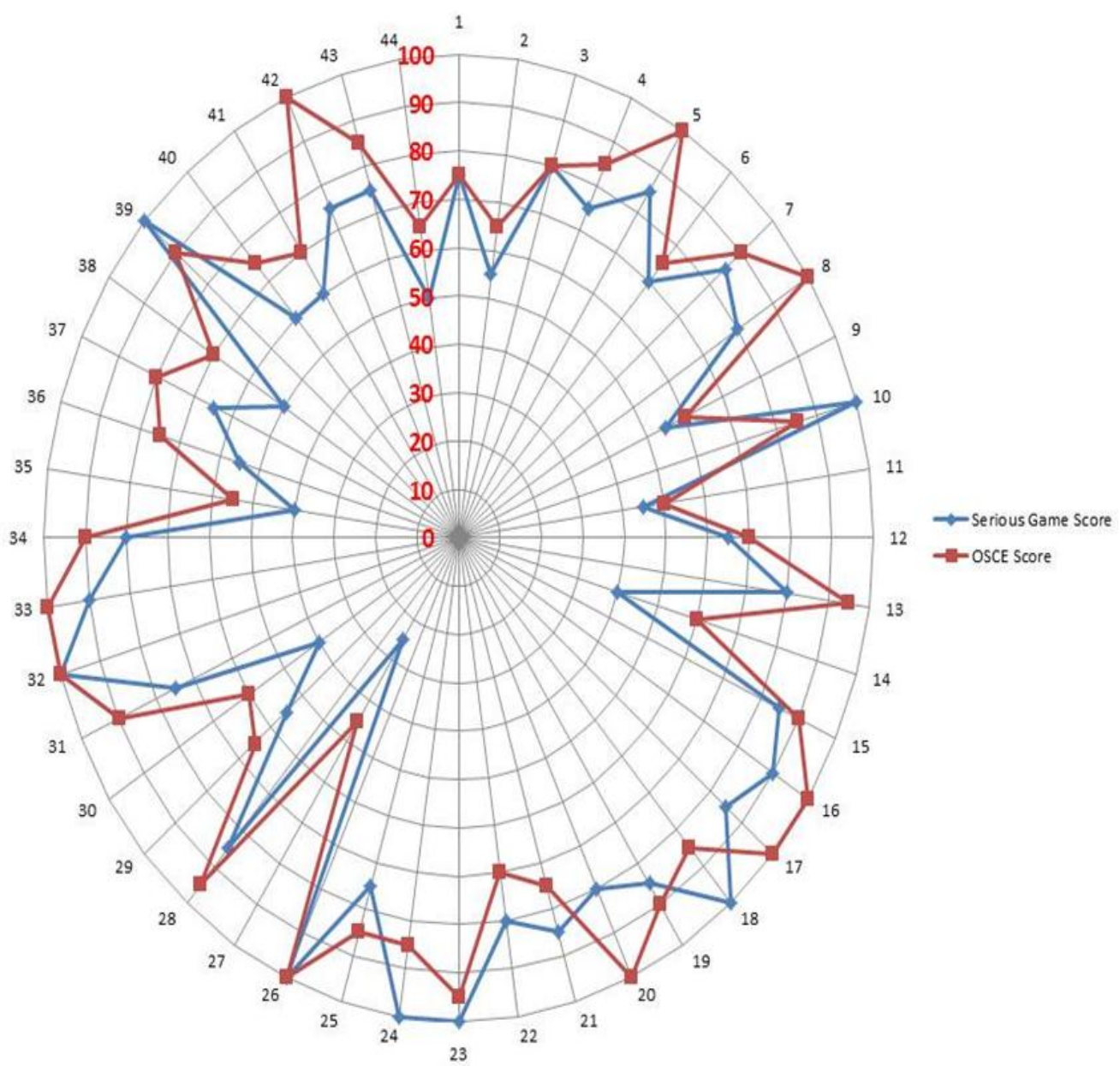

Figure-3. Correlation between the investigation group participants' serious game test results and OSCE scores. Source: CASE-Acibadem University -diagram comparing each participants' serious game test result and his/her OSCE score.

\section{Discussion}

Charles P. Friedman suggested that medical education had become 'stuck' in space, time, and content (Friedman, 2009). Kron investigated medical students experience and attitudes towards video games as an educational tool and concluded that medical student respondents, including many of whom do not play video games, held highly favourable views about the use of video games and related to new media technology in medical education (Kron, Gjerde, Sen, \& Fetters, 2010).

There are several former studies comparing computer-based education tools and traditional learning in medical education, but the results of these studies were rather controversial. Goldberg and Mc Khann investigated the performance of students learning neuroscience in a virtual learning environment with those learning the same topic presented in conventional lectures. They concluded that students who learned in the virtual environment had significantly higher test scores (Goldberg \& McKhann, 2000). Rondon, Sassi and de Andrada, on the other hand, compared the effects of game-based education to traditional learning on students' knowledge retention of head and neck Anatomy and Physiology. Their results demonstrated that game based education shows comparable efficiency in short term gains, but that traditional learning is more effective in improving students' short and long term knowledge retention (Rondon, Sassi, \& de Andrade, 2013). A study on Basic Life Support concluded that self-learning station video training can introduce CPR skills, but additional voice feedback exercises are needed to achieve acceptable CPR quality (Mpotos et al., 2013).

According to our findings, serious gaming is suitable as a teaching strategy for CPR training. Our study suggests that computer-based and traditional learning is comparable in terms of learning efficiency. In other words, students in the intervention group who completed their BLS education at home showed comparable learning performance and OSCE results to the control group who spent 2 hours with the faculty. Therefore, the results suggest that web-based learning models provide an effective learning experience for students and teachers, which can reduce faculty teaching hours in the overloaded curriculum of medical students.

We also concluded that web-based learning models reduce training costs, facilitate the students' access to repeated information, suit the learners' personal needs, influence the standardization of the available information, and give students the opportunity to learn in a convenient way at their own pace and place. This opportunity eased students' learning process to acquire the presented knowledge 
And since skill decay is an often observed problem in BLS training, another advantage of serious games as an educational tool is their repeatable nature without reliance on additional faculty time. Our secondary result, that the automated serious game test scores of participants in the investigation group were very similar to their OSCE scores implies that serious gaming could not only be an additional tool for teaching but also be useful in the assessment of the medical students.

\section{Limitations}

There were two limitations in the development phase of our serious game module that hindered the workflow; network connectivity problems and software bugs. In the development phase of our study, two serious game versions were produced, albeit with errors. In the last and the third version, the aforementioned problems were solved. Participants who attended the last version, which we used in our study, reported no technical problems with the serious game.

A limitation of our study design was the small sample size. Future work will address this problem by recruiting more participants. Another limitation of our study design was participation bias. All participants in our sample were medical students. Since BLS training is essential for almost everybody, future work will investigate the efficiency of our serious game module on non-medical students as well.

\section{Conclusion}

Extrapolating on the current evidence, we conclude that the use of serious games as a self-learning strategy before hands-on training will be beneficial as it can reduce training costs and faculty time while showing comparable efficiency as traditional teaching modalities. These results implicate serious games as a novel teaching modality that suit the learning styles of our students, thus further encourage us to work on creating serious games for other learning objectives.

\section{References}

Baptista, A. V. (2011). Non-traditional adult students: Reflecting about their characteristics and possible implications for higher education. Procedia -Social and Behavioral Sciences, 30, 752- 756 . Available at: 10.1016/j.sbspro.2011.10.147.

Dennen, V. P., \& Burner, K. J. (2008). The cognitive apprenticeship model in educational practice. Handbook of Research on Educational Communications and Technology, 3, 425-439.

Friedman, C. P. (2009). The marvelous medical education machine or how medical education can be 'unstuck' in time. Medical Teacher, 22(5), 496-502. Available at: 10.1080/01421590050110786.

Goldberg, H. R., \& McKhann, G. M. (2000). Student test scores are improved in a virtual learning environment. Advances in Physiology Education, 23(1), S59-66. Available at: 10.1152/advances.2000.23.1.S59.

Halachev, P. M. (2009). E-learning effectiveness. Paper presented at the In Interactive Computer Aided Learning International Conference Kessel University Press, Austria.

Holzinger, A. (2008). HCI and usability for education and work 4th Symposium of the Workgroup Human-Computer Interaction and Usability Engineering of the Austrian Computer Society, USAB 2008, Graz, Austria, November 20-21, 2008, Proceedings. Berlin: Springer Verlag.

Kron, F. W., Gjerde, C. L., Sen, A., \& Fetters, M. D. (2010). Medical student attitudes toward video games and related new media technologies in medical education. BMC Medical Education, 1O(1), 50. Available at: 10.1186/1472-6920-1050 .

Maloney, S., Nicklen, P., Rivers, G., Foo, J., Ooi, Y. Y., Reeves, S., \& Ilic, D. (2015). A cost-effectiveness analysis of blended versus face-to-face delivery of evidence-based medicine to medical students. Journal of Medical Internet Research, 17(7), e182. Available at: 10.2196/jmir.4346.

Mpotos, N., De Wever, B., Calle, P. A., Valcke, M. A., Peersman, W., \& Monsieurs, K. G. (2013). Acquiring basic life support skills in a self-learning station. European Journal of Emergency Medicine, 20(5), 315-321. Available at: 10.1097/MEJ.ob013e328358490a.

Papastergiou, M. (2009). Digital game-based learning in high school computer science education: Impact on educational effectiveness and student motivation. Computers $\mathcal{E}^{\circ}$ Education, 52(1), 1-12. Available at: 10.1016/j.compedu.2008.06.004.

Perkins, G. D., Handley, A. J., Koster, R. W., Castrén, M., Smyth, M. A., Olasveengen, T., \& Ristagno, G. (2015). European resuscitation council guidelines for resuscitation 2015: Section 2.Adult basic life support and automated external defibrillation. Resuscitation, 95, 81-99. Available at: 10.1016/j.resuscitation.2015.07.015.

Prensky, M. (2012). From digital natives to digital wisdom: Hopeful essays for 21 st century learning. Thousand Oaks, Calif: Corwin Press.

Rondon, S., Sassi, F. C., \& de Andrade, C. R. F. (2013). Computer game-based and traditional learning method: A comparison regarding students' knowledge retention. BMC Medical Education, 13(1), 30. Available at: 10.1186/1472-6920-13-30.

Ruiz, J. G., Mintzer, M. J., \& Leipzig, R. M. (2006). The impact of e-learning in medical education. Academic Medicine, 81(3), 207-2 12. Available at: 10.1097/00001888-200603000-00002

Saini, K., \& Wahid, D. A. (2014). Traditional learning versus web based learning: Performance analysis. International Journal of Computer Science and Information Technologies, 5(4), 5182-5184.

Vygotsky, L. S. (1978). Mind in society: The development of higher psychological processes. Cambridge. Mass: Harvard University Press. 
Walz, S. P., \& Deterding, S. (2014). The gameful world: Approaches, issues, applications. Cambridge, Massachusetts; London. England: The MIT Press.

Wilson, L., \& Wittmann-Price, R. A. (2015). Review manual for the certified healthcare simulation educator (CHSE) exam. NYC: Springer Publishing.

Yolton, R. L., \& Calesta, D. (2000). Pacific's experience with web-based instruction: Bats in the belfry or webs in the classroom? Optometry, $71(1)$, 20-28. 